\title{
The Fall Speed Variability of Similarly Sized Ice Particle Aggregates
}

\author{
CARl G. SCHMitT, ${ }^{\mathrm{a}}$ KARA Sulia, ${ }^{\mathrm{b}}$ ZACHARY J. Lebo,${ }^{\mathrm{c}}$ ANDREW J. HeYMSFIELD, ${ }^{\mathrm{a}}$ \\ VANESSA PRZYByo, ${ }^{\mathrm{b}}$ AND PAUl CONNOLLY ${ }^{\mathrm{d}}$ \\ ${ }^{a}$ National Center for Atmospheric Research, Boulder, Colorado \\ ${ }^{\mathrm{b}}$ Atmospheric Sciences Research Center, University at Albany, State University of New York, Albany, New York \\ ${ }^{\mathrm{c}}$ University of Wyoming, Laramie, Wyoming \\ ${ }^{\mathrm{d}}$ School for Earth and Environmental Sciences, University of Manchester, Manchester, United Kingdom
}

(Manuscript received 2 November 2018, in final form 22 April 2019)

\begin{abstract}
The terminal velocity $\left(V_{t}\right)$ of ice hydrometeors is of high importance to atmospheric modeling. $V_{t}$ is governed by the physical characteristics of a hydrometeor, including mass and projected area, as well as environmental conditions. When liquid hydrometeors coalesce to form larger hydrometeors, the resulting hydrometeor can readily be characterized by its spherical or near-spherical shape. For ice hydrometeors, it is more complicated because of the variability of ice shapes possible in the atmosphere as well as the inherent randomness in the aggregation process, which leads to highly variable characteristics. The abundance of atmospheric processes affecting ice particle dimensional characteristics creates potential for highly variable $V_{t}$ for ice particles that are predicted or measured to be of the "same size." In this article we explore the variability of ice hydrometeor $V_{t}$ both theoretically and through the use of experimental observations. Theoretically, the variability in $V_{t}$ is investigated by analyzing the microphysical characteristics of randomly aggregated hexagonal shapes. The modeled dimensional characteristics are then compared to aircraft probe measurements to constrain the variability in atmospheric ice hydrometeor $V_{t}$. Results show that the spread in $V_{t}$ can be represented with Gaussian distributions relative to a mean. Variability expressed as the full width at half maximum of the normalized Gaussian probability distribution function is around $20 \%$, with somewhat higher values associated with larger particle sizes and warmer temperatures. Field campaigns where mostly convective clouds were sampled displayed low variability, while Arctic and midlatitude winter campaigns showed broader $V_{t}$ spectra.
\end{abstract}

\section{Introduction}

Observations regularly show a myriad of ice particle shapes within an ice cloud, even when only particles within a narrow size range are considered. The ice microphysical research community has adopted a onedimensional unit of measure (length, or maximum dimension) to categorize atmospheric ice particles. This leads to significant, generally uncharacterized, uncertainty given that atmospheric ice particles are three-dimensional while measurements are generally two-dimensional images of the ice particles. The onedimensional length is extracted from the two furthest points observed on a two-dimensional image of the particle or by determining the diameter of the smallest circle that will encompass the particle image. Despite the possible variability in the images, the maximum

Corresponding author: Carl G. Schmitt, schmittc@ucar.edu measured lengths are "binned" to populate an ice particle size distribution (PSD). Because this process involves the determination of a size of a threedimensional irregular object through the analysis of a two-dimensional image of that object, inherent uncertainty exists in the shapes and sizes of the individual bins as well as the resulting PSD (Wu and McFarquhar 2016).

Aircraft measurements of ice particles are also affected by commonly ignored effects. For example, image data are typically collected for one orientation and the true maximum dimension is not guaranteed to be imaged thus leading to uncertainty. Further, an aircraft probe that can measure ice particle mass has yet to be developed. Important constraints can be put on ice particle mass through the use of total ice water content measurements such as the counterflow virtual impactor (CVI; Twohy et al. 1997). Some newer aircraft probes, including the Stratton Park Engineering Company Inc. 
(SPEC Inc.) two-dimensional stereo probe (2DS; Lawson et al. 2006) and the Karlsruhe Institute of Technology Particle Habit Imaging and Polar Scattering probe (PHIPS; Abdelmonem et al. 2011), provide two images of each particle, improving our understanding of the characteristics.

The dimensional characteristics of atmospheric ice particles are important for many aspects of weather and climate modeling. The exchange of water in all phases from different levels through the sedimentation and advection of ice particles requires an understanding of the mass of the individual particles and ice particle terminal velocity $\left(V_{t}\right)$. Ice shape and particle projected area affect electromagnetic radiation directly, thus impacting the energy balance. Both ice particle mass and projected area are directly related to $V_{t}$ of ice particles, which secondarily impacts both mass exchange and radiation transfer. Particle growth and evolution of aspect ratio (size and shape) through vapor diffusion is primarily controlled by air temperature, while growth can also occur through aggregation of crystals and riming. For individual crystals growing from vapor alone, the Adaptive Habit Model (Harrington et al. 2013a,b; Sulia et al. 2013) predicts the growth of hexagonal crystal axes based on temperature. Hence, ice crystals measured within a given size bin can be affected by all of these processes to varying degrees, leading to a diverse population of particle characteristics in each size bin.

$V_{t}$ is mainly a function of particle projected area and mass (Heymsfield and Westbrook 2010), which have been studied extensively (Brown and Francis 1995; Mitchell 1996; Heymsfield and Miloshevich 2003; Heymsfield et al. 2004, 2011, 2013). Most of these studies assign one mass and one projected area per ice particle size, and the area-dimensional relationships are not mathematically linked to the mass-dimensional relationships. By studying the fractal characteristics of aggregates of ice particles, Schmitt and Heymsfield (2010, hereafter, SH10) were able to directly relate particle area-dimensional relationships to massdimensional relationships. The SH10 relationships are valid when ice particles are fractal in nature, which tends to occur at particle sizes larger than approximately 300-750 $\mu \mathrm{m}$ (Schmitt and Heymsfield 2014; Schmitt et al. 2016), depending on the ambient conditions. Fractal particles grow randomly, often through aggregation. The dimensional characteristics of fractal particles can be expressed as power-law relationships with the power being the fractal dimension. For standard power-law mass- and area-dimensional relationships, the fractal dimension is simply the power that $D$, the particle maximum dimension, is raised with the two-dimensional fractal dimension being the power in area relationships while the three-dimensional fractal dimension is the power in mass relationships. SH10 describes the relationship between the two-dimensional fractal dimension and the three-dimensional fractal dimension as they pertain to atmospheric ice particles. By assuming that atmospheric ice particles are fractal or quasi-fractal, it is possible to relate their mass and projected area, presumably leading to more realistic $V_{t}$ estimates.

A major flaw in parameterizing ice crystal dimensional characteristics in numerical models using massand area-dimensional relationships is that unless the relationships are developed with the understanding that these characteristics are related, the resulting ice particle characteristics could be unphysical, often affecting very large or very small particles substantially. The lack of a physical connection among these particle characteristics deems the model microphysics uncertain, where these uncertainties in particle shape and size lead to uncertainties compound in other microphysical processes, such as collection, sedimentation, and consequently the overall mass and energy budgets of the system. Removing the arbitrary nature of estimates of mass- and area-dimensional relationship measurements and instead mathematically linking them together provides for a stronger physical connection among model-derived particle characteristics, which is expected to improve the accuracy of microphysical parameterizations.

Previous research by Sasyo and Matsuo (1980) has shown that snow measured at the surface falls with a Gaussian fall speed distribution centered around $100 \mathrm{~cm} \mathrm{~s}^{-1}$ with a roughly constant $12 \mathrm{~cm} \mathrm{~s}^{-1}$ standard deviation regardless of mass. Passarelli and Srivastava (1979) showed that when compared to particles of similar mass, the variability in fall speed could exceed plus or minus $25 \%$. Sasyo and Matsuo (1985) showed that fall speed variability could reduce considerably the time required to reach a specific snowfall rate when compared with a collision kernel that did not include variable terminal velocity for similar sized snow. While these observations were for snow particle sizes much larger (multiple millimeters to centimeters) than in the current study (maximum $1 \mathrm{~mm}$ ), but demonstrate similar variability in the distribution around the average as well as the importance of considering the variability of fall speed within a particle population.

In this article, we explore the variability in dimensional characteristics possible within a measured size bin. Using theoretically generated aggregates as well as aircraft measurements, we develop estimates of the extent of possible variability in $V_{t}$ for a given size. 
These results are useful for applications involving differential fall speed for aggregation as well as for bin microphysics modeling in general by adding variability to the relationship between particle $V_{t}$ and size. In section 2 , the estimates using theoretically created aggregates from an ice particle aggregate simulator (IPAS) will be discussed. In section 3, the methodology for applying these estimates to aircraft data are discussed. Section 4 presents results from the SPEC Inc. cloud particle imager probe (CPI; Lawson et al. 2001) from several field campaigns. Section 5 discusses the relationships between the IPAS and CPI studies and uncertainties that exist between the datasets. In section 6 , key points of the research are summarized and conclusions and limitations are discussed.

\section{Simulated particles}

To quantify the variability of $V_{t}$ within a single size bin, it is necessary to understand the variability of the projected area and mass of the particles within that size bin. It is incomplete to simply look at raw measurements because tools exist to investigate theoretically the relationship between three-dimensional structure and two-dimensional images and therefore reduce uncertainty between related particle characteristics. To address these uncertainties, we use the IPAS (SH10; Schmitt and Heymsfield 2014; note that the model was not referred to as IPAS in the previous publications). For a detailed description of the IPAS model, the reader is directed to Przybylo et al. (2019).

IPAS has been used to develop a dataset of theoretical individual ice particles (elementary crystals) and aggregates of ice particles. IPAS uses hexagonally shaped elementary crystals with a defined aspect ratio to randomly build aggregates. IPAS was developed to simulate measurements of atmospheric ice particles imaged by aircraft probes and can be configured to study different viewing angles. Because IPAS creates theoretical aggregates of ice particles by adding elementary crystals to an aggregate, it is possible to determine the exact mass and projected area for $V_{t}$ calculations when a particle size is assumed. The variability of these factors is a result of the randomness inherent in the aggregation process employed by IPAS. For this study, 10 different aspect ratios of hexagons ranging from 0.2 to 5.0 (plates to columns, aspect ratio $=$ length along prism face/length across hexagonal face) were used to generate theoretical ice crystal aggregates of up to 10 elementary crystals. For simplicity, the maximum dimension of each of the elementary crystals was normalized to $50 \mu \mathrm{m}$, then 200 theoretical aggregates were generated. Measurements

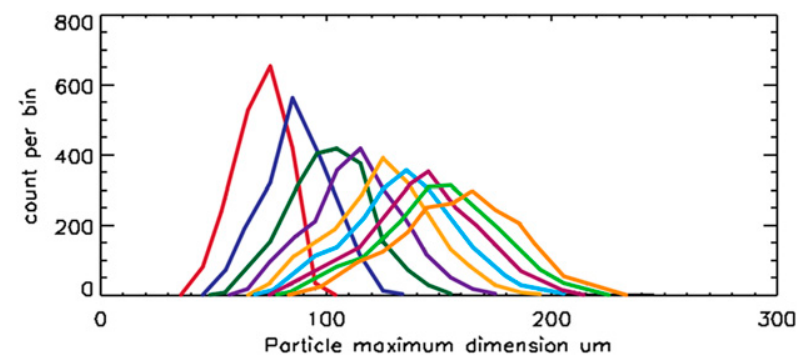

FIG. 1. Ten curves showing the variability in measured maximum dimension for IPAS particles. Different colors represent different numbers of elementary crystals (increasing from left to right) in the theoretical aggregate (e.g., 2 elementary crystals: red, 10 elementary crystals: dark orange).

of the aggregate dimensional characteristics from several theoretical viewing angles were made after each elementary crystal was attached to the aggregate resulting in approximately 20000 sets of particle characteristics for comparison with aircraft-measured microphysical parameters. For the IPAS calculations, the theoretical aggregates were oriented into the expected fall orientation, with the maximum projected area perpendicular to the falling direction (Cho et al. 1981).

Combining the data from all aspect ratios, Fig. 1 shows the size range of the generated theoretical aggregates with different numbers of elementary crystals. Each curve represents a size distribution for the theoretical aggregates with the given number of elementary crystals for all aspect ratios (2 elementary crystals for the leftmost distribution and 10 elementary crystals for the rightmost). As might be expected, the aggregates of two elementary crystals had sizes that ranged from $\sim 50$ (the size of an individual elementary crystal) to $100 \mu \mathrm{m}$ (the size of two elementary crystals lined up end to end). Aggregates of 10 elementary crystals had a large range of possible sizes, that is, from approximately 80 to nearly $250 \mu \mathrm{m}$. Note that the $90-100-\mu \mathrm{m}$ size bin could include particles with as few as 2 and as many as 10 elementary crystals. One would expect that this could lead to a significant range in potential $V_{t}$ values for particles classified as being the same size.

For given microphysical parameters, it is possible to estimate $V_{t}$ using Heymsfield and Westbrook (2010). The Heymsfield and Westbrook (2010) formulation was developed using experimental data and includes a correction for particle projected area, which earlier studies did not include. For the IPAS dataset, $V_{t}$ was calculated for each particle and then compared to the mean $V_{t}$ for all particles within $10-\mu \mathrm{m}$ size bins collectively and for each aspect ratio separately. Note that for all $V_{t}$ calculations, the data were normalized to a temperature of $0^{\circ} \mathrm{C}$ and a pressure of $1000 \mathrm{hPa}$ for consistency. 

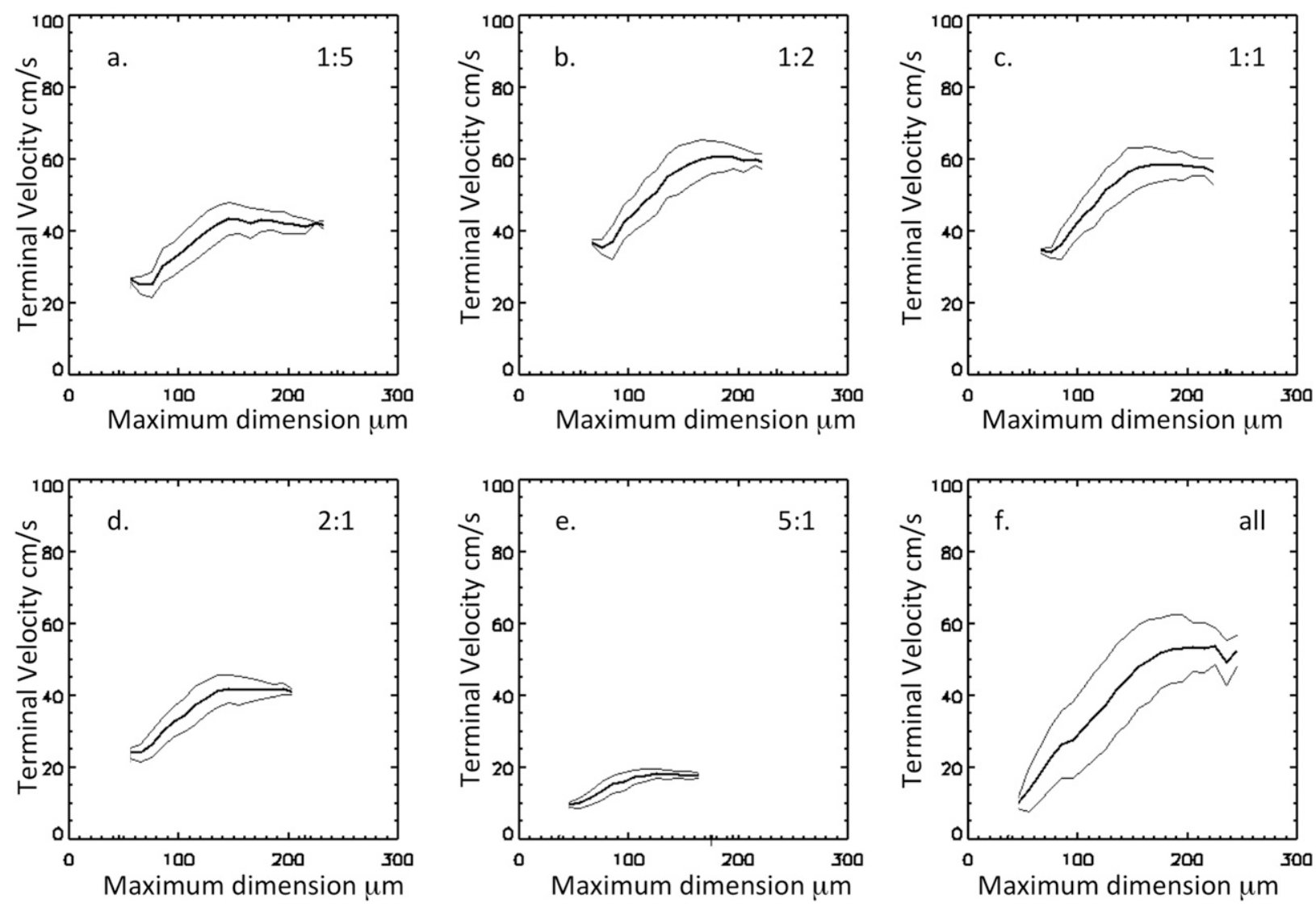

FIG. 2. Terminal velocity $V_{t}$ by particle size for selected IPAS elementary crystal aspect ratios. The bold line is the median $V_{t}$ value for each $10-\mu \mathrm{m}$ size bin; the thin lines representing \pm one standard deviation. Data are plotted for 200 aggregates each with aspect ratios of (a) $1: 5$, (b) 1:2, (c) 1:1, (d) 2:1, (e) 5:1, and (f) the full dataset with all aspect ratios combined.

For each aspect ratio subset of the data, the $V_{t}$ to size relationship is shown in Fig. 2. In each panel, the median $V_{t}$ in each $10-\mu \mathrm{m}$ size bin is shown as well as \pm one standard deviation. Note that the standard deviation is a relatively constant $20 \%-30 \%$ of the median $V_{t}$ value for single aspect ratio panels, although it is higher when all aspect ratios are considered together. For the individual aspect ratios (Figs. 2a-e), the values begin to flatten out at larger sizes, perhaps suggesting that a relatively constant area-to-mass ratio is reached for the larger aggregates. In Fig. 2f (also Figs. 2a-e to a lesser extent), the reduction in uncertainty at sizes larger than $180 \mu \mathrm{m}$ is unsurprising when the data presented in Fig. 1 are considered. For particles larger than $180 \mu \mathrm{m}$, most of the particles have high elementary crystal numbers, whereas for aggregates in the middle of the size range, a larger variety of elementary crystal numbers is present in each size bin. Thus, the area-to-mass relationship is being artificially constrained at larger and smaller sizes, reducing the apparent variability. Moreover, the high aspect ratios for columns (Fig. 2e) tend to be smaller in overall size compared to the other aspect ratios. It is anticipated that for a dataset including aggregates with much higher numbers of elementary crystals, the variability observed in the middle of the ranges would continue to much larger sizes.

For modeling efforts using techniques such as the Adaptive Habit Model, where vapor growth parameters are controlled by atmospheric conditions (e.g., Harrington et al. 2013a,b; Sulia et al. 2013; Jensen et al. 2017), these results suggest that even when aggregation is considered, a relationship between vapor-grown ice crystal aspect ratio and $V_{t}$ will be useful given the impact of elementary crystal characteristics on the resulting aggregate. For example, for a given aspect ratio and elementary crystal count, the terminal velocities are very similar (Fig. 3), which is a result of a lack of variability in particle projected area for a given elementary crystal count and aspect ratio. As the elementary crystal count increases, the mass increases linearly, and the projected area increases at a slightly less than linear rate; thus, the average mass-to-area ratio increases slowly, leading to increased $V_{t}$. Hence, the variability present in each size bin in Figs. 2a-e 

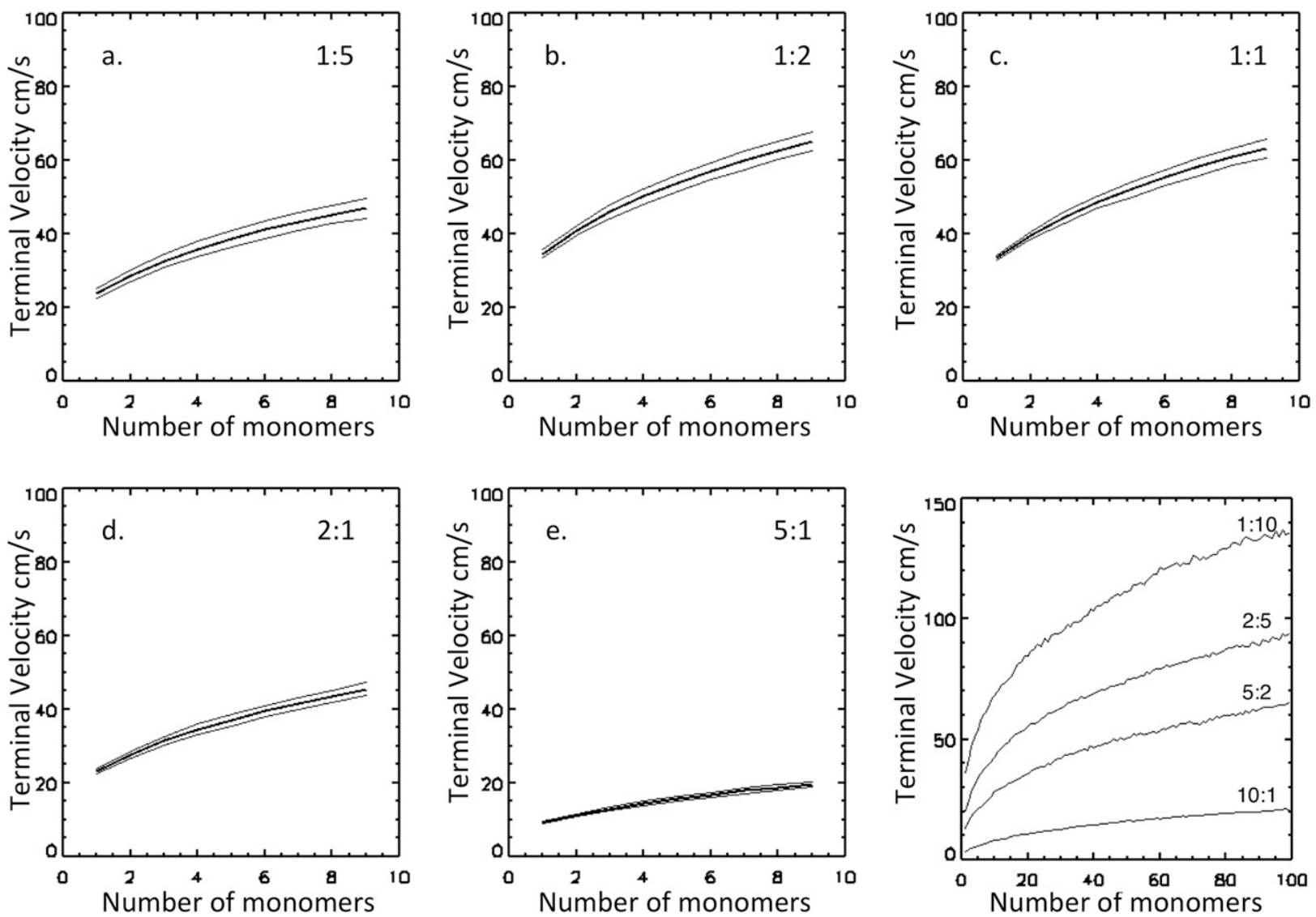

FIG. 3. Mean and \pm one standard deviation of $V_{t}$ calculated for theoretical aggregates of 1-9 elementary crystals of different aspect ratios; (a)-(e) same aspect ratios as Fig. 2 (20 aggregates for each plotted values), and (f) mean $V_{t}$ calculated for up to 100 elementary crystals of 4 different aspect ratios (4 aggregates per plotted value).

is more likely attributed to the elementary crystal count, whereas for Fig. 2f, the variability can be attributed to the elementary crystal aspect ratio. Figure $3 \mathrm{f}$ shows the mean $V_{t}$ calculated for aggregates of up to 100 elementary crystals created with four different aspect ratio elementary crystals $(1: 10,2: 5,5: 2$, and 10:1). After an initial increase in $V_{t}$, the increase is moderated. Thus, for an Adaptive Habit Model type scheme in which aspect ratio (elementary crystal and aggregate) is predicted, $V_{t}$ can be better estimated for aggregates using the aspect ratio and number of elementary crystals rather than the more complicated process of extracting the particle dimensions.

\section{Aircraft measurement methodology}

The SPEC Inc. CPI probe collects high-resolution images $(2.3 \mu \mathrm{m}$ per pixel) of ice particles and is ideally suited for the study of the variability of shape of ice particles larger than $50 \mu \mathrm{m}$. Using CPI data, a large dataset from 10 field campaigns conducted globally was analyzed to investigate the variability of several ice particle parameters related to $V_{t}$, including particle maximum dimension, particle projected area, and area ratio (the ratio of the area of the particle to the area of the smallest encompassing circle). Table 1 provides a summary of the field campaign data that were used for this project. The parameters extracted from the CPI data were used to estimate the variability in $V_{t}$. Particle images were only included in the study if they were the only particle in the CPI frame, reducing the likelihood of shattered particles being included. Moreover, out of focus particles were rejected by selecting particles with focus values (as determined by CPIview software) of 45 or higher. Particles that touched the edge of the field of view were also excluded (McFarquhar et al. 2013).

Using the fractal relationships in SH10, the mass and projected area of fractal particles can be related. Fractal particles can be segregated from nonfractal particles by the complexity $(C)$ value defined in Schmitt and Heymsfield (2014). For the purpose of this research, 
TABLE 1. Table of data used. Research acronyms, location, dates, and regional classifications are included as well as a reference for each program for further information. ACCACIA: Aerosol Cloud Coupling and Climate Interactions in the Arctic project; MPACE: Mixed Phase Arctic Cloud Experiment; ARM-IOP: Department of Energy Atmospheric Radiation Measurement Intensive Operating Period at the Southern Great Plains site; MidCiX: Midlatitude Cirrus Experiment; EMERALD: Egrett Microphysics Experiment with Radiation, Lidar, and Dynamics; AIRS II: Alliance Icing Research Study II; ICE-L: Ice in Clouds Experiment-Layers; ACTIVE: Aerosol and Chemical Transport in Deep Convection project; CRYSTAL-FACE: Cirrus Regional Study of Tropical Anvils and Cirrus LayersFlorida-Area Cirrus Experiment; ICE-T: Ice in Clouds Experiment-Tropical.

\begin{tabular}{lllll}
\hline \hline \multicolumn{1}{c}{ Campaign } & \multicolumn{1}{c}{ Location } & \multicolumn{1}{c}{ Date } & Region & \multicolumn{1}{c}{ Reference } \\
\hline ACCACIA & Svalbard & Mar-Apr 2013 & Arctic & Lloyd et al. (2015) \\
MPACE & Alaska & Sep-Oct 2004 & Arctic & Verlinde et al. (2007) \\
ARM-IOP & Oklahoma & Mar 2000 & Midlatitude & Heymsfield et al. (2002) \\
MidCix & Western United States & May 2004 & Midlatitude & Heymsfield et al. (2006) \\
EMERALD & Adelaide, Australia & Sep 2001 & Midlatitude & Whiteway et al. (2004) \\
AIRS-II & Quebec, Canada & Nov 2003-Feb 2004 & Frontal & Isaac et al. (2005) \\
ICE-L & Colorado & Nov 2007 & Frontal & Heymsfield et al. (2011) \\
ACTIVE & Darwin, Australia & Nov 2005-Feb 2006 & Tropical & Vaughan et al. (2008) \\
CRYSTAL-FACE & Florida & Jun, Jul 2002 & Tropical & Jensen et al. (2004) \\
ICE-T & U.S. Virgin Islands & Jul 2011 & Tropical & Heymsfield and Willis (2014) \\
\hline
\end{tabular}

particles with $C$ values lower than 0.45 were removed from the study. While 0.45 is in the transition zone between nonfractal and fractal (Schmitt et al. 2016), the results of the previous section suggest that transition particles are reasonable to consider (in Fig. 3 there is not a transition at any stage that suggests that the nonfractal to fractal transition is particularly special. The transition from nonfractal to fractal would occur in the 3-6 elementary particle range.) Mass- and area-dimensional relationships can be related for fractal particles as follows, respectively:

$$
\begin{aligned}
& m=a D^{b}, \quad \text { and } \\
& A=c D^{d},
\end{aligned}
$$

where $m$ is particle mass, $D$ is particle maximum dimension, $a$ and $c$ are the fractal prefactors, and $b$ and $d$ are the three- and two-dimensional fractal dimensions.

From SH10, we know that $b=1.3 \times d$. Note that SH10 showed that the 1.3 value stated above can vary from 1.23 to 1.33 depending on the aspect ratio of the elementary crystals that make up the aggregate. Here we use 1.3 as it is anticipated to be more representative of natural ice particles as the lowest values were more commonly associated with lower area ratio aggregates not frequently seen in the atmosphere. The area ratio distribution observed for the aggregates composed of elementary crystals with aspect ratio 2.0 or lower were in better agreement with the area ratio distributions observed in the field program data as well as the area ratio values reported in Korolev and Isaac (2003). Inserting $1.3 d$ into Eq. (2) gives

$$
m=\frac{a}{c} c D^{d} D^{0.3 d}
$$

by substituting and breaking up the power and multiplying by $c / c$; thus, $A$ can be substituted into Eq. (3), which leads to

$$
m=\frac{a}{c} A D^{0.3 d} .
$$

Thus, for a population of fractal particles with the same maximum dimension (within the same size bin), it can be assumed that the mass is linearly proportional to the particle projected area or area ratio.

Because the purpose of this article is to investigate the variability in $V_{t}$ within a size bin, the relationship in Eq. (4) can be used to adjust the mass of individual particles around assumed mean values. The mass adjustment for an individual particle is made (as dictated by the fractal relationship) in proportion to the difference in the projected area of the particle from the mean value of the projected area of all particles within the size bin. This assumes that the particles are fractal or near fractal and is assured by the complexity cutoff mentioned earlier. Because the actual mass of individual ice particles measured by aircraft probes is unknown, the average particle mass for a size bin was assumed to be the mass calculated using the relationship developed in Heymsfield et al. (2004). It was found that the selection of mass-dimensional relationships had very little effect on the results of the study, thus Heymsfield et al. (2004) was used for all datasets. This mass value for a particular particle size was then adjusted based on the ratio of the projected area divided by the average area for all particles in the size bin $m_{\text {new }}=m_{\text {calc }} \times$ $\left(a_{\text {particle }} / a_{\text {average }}\right)$. Then, for each individual particle, the measured projected area and the newly estimated mass were used to determine a unique $V_{t}$ for each particle using Heymsfield and Westbrook (2010). 

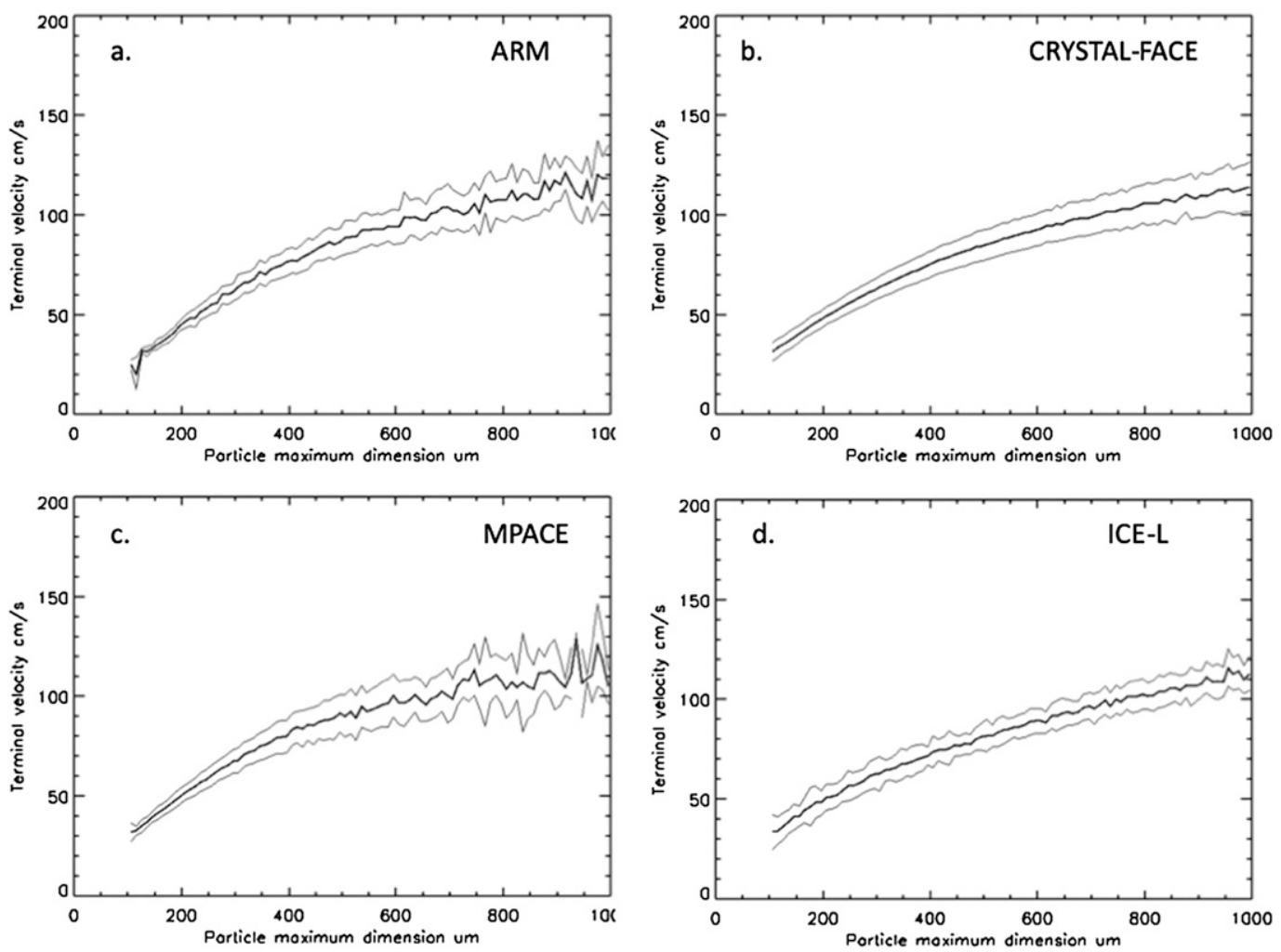

FIG. 4. $V_{t}$ median and \pm one standard deviation spread estimated for CPI particles in four field campaigns in varying locations globally. The larger spread shown for MPACE is likely due to frequent occurrences of large columns as well as graupel, which lead to a wide range of area ratio values.

This procedure results in an ensemble of $V_{t}$ values for each size bin. Probability distribution functions (PDFs) for a selection of particles were determined by dividing the estimated $V_{t}$ [from Eq. (4)] by the average $V_{t}$ value estimated (i.e., if the $V_{t}$ value of a particle was determined to be $30 \mathrm{~cm} \mathrm{~s}^{-1}$ while the average for the size was $25 \mathrm{~cm} \mathrm{~s}^{-1}$, the values used for the PDF would be 1.2).

\section{Results from aircraft measurements}

Figure 4 shows the mean and \pm one standard deviation of the estimated $V_{t}$ values for observed CPI particles from four example field campaigns. Aside from the Mixed-Phase Arctic Cloud Experiment (MPACE), the datasets all show relatively tight standard deviation envelopes, which is likely because the particles are more fractal in the Atmospheric Radiation Measurement Program intensive observational period (ARM-IOP), CRYSTAL-FACE, and Ice in Clouds Experiment-Layer Clouds (ICE-L) field campaigns, whereas during MPACE, larger lessfractal particles were more common (large needles and early aggregates of needles). Note that in Schmitt et al. (2016), the Arctic datasets (including MPACE) contained some of the largest "single" crystals at warmer temperatures $\left(T>-20^{\circ} \mathrm{C}\right)$ when compared to observations in other regions. Much of the data collected during MPACE were in warmer mixed-phase clouds, where large needles as well as heavily rimed graupel particles were common and thus influenced the spread of estimated $V_{t}$ values. The ARM-IOP data included several flights where the clouds contained mostly bullet rosette-shaped crystals; additionally, some convective cloud measurements were collected. Thus, the variety of observed shapes likely increased the spread in comparison to CRYSTAL-FACE and ICE-L, which included very active frontal and convective clouds that were likely fractal in nature.

In most cases, $V_{t}$ variability appears to be normally distributed around the mean and thus were fit to Gaussian functions of the form

$$
V_{t}=A_{0} e^{-z^{2} / 2} \quad \text { where } \quad z=\frac{x-A_{1}}{A_{2}}
$$

$A_{0}$ is the height of the Gaussian, $A_{1}$ is the center, and $A_{2}$ is the Gaussian width, which is also the standard deviation. The full width at half maximum [the width of 
TABLE 2. For each field campaign, overall statistics of $V_{t}$ variability including the standard deviation of the projected area (and mass, assuming fractal properties) and the FWHM of the Gaussian fit to the normalized $V_{t}$ values.

\begin{tabular}{lcc}
\hline \hline Field campaign & $\begin{array}{c}\text { Standard deviation \% of } \\
\text { normalized projected } \\
\text { area }(100-1000 \mu \mathrm{m})\end{array}$ & $\begin{array}{c}\text { FWHM for } \\
\text { normalized Gaussian } \\
\text { distribution of } V_{t}\end{array}$ \\
\hline ARM-IOP & 25.2 & 0.2441 \\
ACTIVE & 21.7 & 0.1634 \\
CRYSTAL- & 24.9 & 0.2197 \\
$\quad$ FACE & & \\
EMERALD & 21.6 & 0.1805 \\
MIDCIX & 18.8 & 0.1569 \\
AIRS2 & 27.2 & 0.2348 \\
CRYSTAL- & 24.2 & 0.2184 \\
$\quad$ FACE WB-57 & & \\
ACCACIA & 30.0 & 0.2637 \\
ICE-L & 22.9 & 0.1875 \\
MPACE & 26.7 & 0.2306 \\
\hline
\end{tabular}

the Gaussian distribution at $50 \%$ of the maximum value (FWHM)] is calculated as

$$
\mathrm{FWHM}=2 \times A_{2} \sqrt{2 \times \log (2)} .
$$

Table 2 lists the FWHM of the spread of $V_{t}$ values around the normalized mean for 10 different field campaigns for particles larger than $100 \mu \mathrm{m}$. Table 2 also shows the standard deviation percentages for the projected area. The variability in projected area is close to a factor of 3 higher than the variability in estimated $V_{t}$. This is due to the use of Eq. (4), which assures that a particle with a smaller area also has a smaller mass. The combined reduction or increase in area coupled with a reduction or increase in mass leads to less variability in $V_{t}$. Note that the standard deviation in the estimated mass (density) would be the same as that for area because of the manner by which mass and area are related. Using different massdimensional relationships or doubling or halving the prefactor only effected the results in Table 2 by $3 \%$ (changing the mass-dimensional relationship) to $5 \%$ [doubling or halving the $a$ parameter in Eq. (1)].

The Arctic field campaigns [Aerosol-Cloud Coupling and Climate Interactions in the Arctic (ACCACIA) and MPACE] as well as the wintertime midlatitude campaign [Alliance Icing Research Study II (AIRS2)] stand out as having moderately higher-than-typical standard deviations, which is likely due to the fact that these datasets included warmer low-altitude measurements where columns, needles, and plate-shaped crystals are more common. Highly convective field campaigns, such as CRYSTAL-FACE and the Aerosol and Chemical Transport in Deep Convection project (ACTIVE), where fractal aggregates were more likely to occur, had lower variability. To further investigate these observations, Fig. 5 shows the FWHM values as a function of temperature for the field campaigns as well as the FWHM for particles separated by size for the field campaigns. For temperatures of approximately $-15^{\circ} \mathrm{C}$ and colder, the FWHM values tend to cluster between 0.175 and 0.25 . However, at higher temperatures, the FWHM values increase significantly (up to 0.3 and 0.4 ) for the low-latitude and wintertime frontal field campaigns (ACCACIA, and AIRS represented with solid lines) implying high variability in $V_{t}$ in each size bin. Conversely, highly convective cases (ACTIVE, CRYSTAL-FACE, and ICE-L) maintain lower $(0.2$ to 0.25$) \mathrm{FWHM}$ values to $0^{\circ} \mathrm{C}$ suggesting less $V_{t}$ variability throughout the column. When the data are viewed with respect to particle sizes, the larger particles show the most variability again, with the low-latitude and wintertime frontal field campaigns standing out compared to the others. Figure 6 shows the PDFs for CRYSTAL-FACE and MPACE for each $100-\mu \mathrm{m}$-size block (offset for comparison purposes), which demonstrates the broader variability as a function of size for MPACE (also represented with a solid line) and the uniformity of CRYSTAL-FACE. Both show $V_{t}$ distributions that are quite Gaussian for the smaller size ranges while for the MPACE data, the Gaussian distribution becomes substantially broader for larger sizes. This is likely due to the more common needle shapes present in warmer $\left(T>-20^{\circ} \mathrm{C}\right)$ mixed-phase conditions that were commonly sampled during MPACE. The very well-mixed convective clouds observed during CRYSTAL-FACE led to very similar in appearance Gaussian distributions across all size ranges.

\section{Synthesis of results and uncertainties}

While the results of the IPAS modeling and the CPI analysis suggest similar results, it is important to fully understand the potential similarities and differences to get the full benefit of this study. The CPI images are categorized by their 2D maximum dimension based on the particle image. As the orientation of particles in the CPI is generally unknown and random orientation is frequently assumed, the maximum dimension of the IPAS particles has been calculated several different ways to quantify its impact on the results. The true maximum dimension of the IPAS particles (calculated by determining the length of the chord from the two farthest separated points in the 3D structure of the aggregate) is on the average $10.5 \%$ larger than the maximum dimension determined from a random $2 \mathrm{D}$ image of the IPAS particle. Recreating Fig. 1 using the maximum dimension from a random $2 \mathrm{D}$ image 

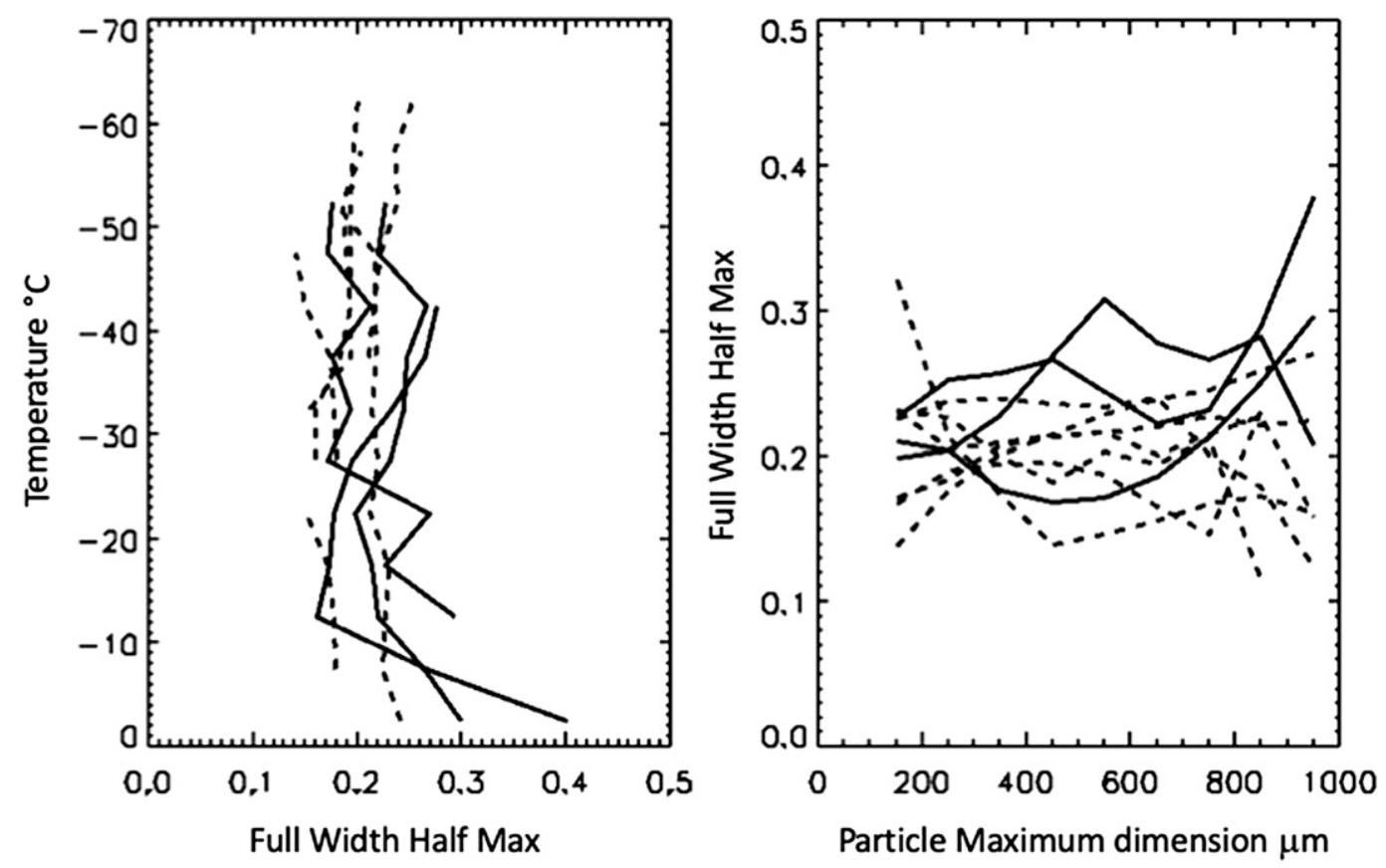

FIG. 5. (left) FWHM for $V_{t}$ variability for different field campaigns plotted by temperature. (right) FWHM of $V_{t}$ variability by particle size. For both presentations, the solid lines indicate data from field campaigns where low temperatures were found at high air pressures (Arctic and winter frontal), whereas the results from field campaigns involving more convective systems (tropical and midlatitude spring-fall) are represented with dashed lines.

showed nearly identical curves, all shifted about $10 \%$ toward smaller sizes. Interestingly, the true maximum dimension is also $2.2 \%$ larger on average than the maximum dimension determined from the orientation with the maximum projected area. The $V_{t}$ values for the individual CPI particles are determined by using the particle projected area or area ratio as compared to average. To determine if the results are comparable between IPAS and the CPI imagery, the IPAS particle area ratio was determined for the anticipated fall orientation as well as for a random orientation. The average of the difference between the two area ratio values was a factor of 1.00087 , suggesting that while the particle maximum dimension and total area may change with orientation, for IPAS particles, the area ratio is invariant with orientation on the average.

While it is critical to understand that the mass adjustments are made by assuming fractal characteristics, the question arises as to whether these results can be applied to less complex particles. Solid convex objects, by definition, have a 2D fractal dimension of 2.0 and a $3 \mathrm{D}$ fractal dimension of 3.0, the variability in shape is based on the prefactor for this type of shape. As noted, the variability in mass-dimensional relationship did not substantially affect the spread in $V_{t}$, nor did the multiplicative $(S)$ factor between used to derive Eq. (3) from Eqs. (1) and (2), thus, the variability in $V_{t}$ is likely similar although the magnitude of the mean $V_{t}$ might be substantially different, although the single elementary crystal values shown in Fig. 3 were in line with the aggregates of 2 or more elementary crystals.

\section{Summary and conclusions}

In this article, the variability in $V_{t}$ has been investigated for ice particles that would be classified to have the

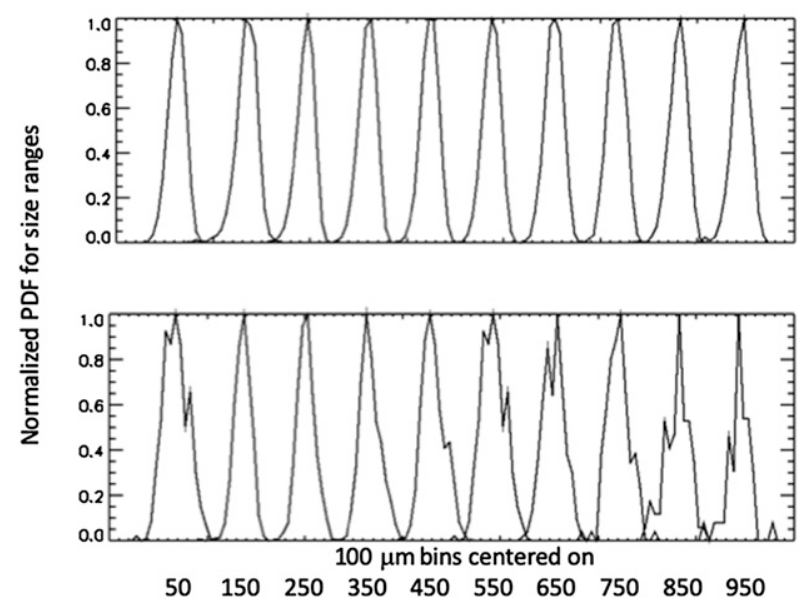

FIG. 6. PDFs of $V_{t}$ distributions by size bin for (top) CRYSTALFACE and (bottom) MPACE for $100-\mu \mathrm{m}$ size bins starting with the $0-100-\mu \mathrm{m}$ size range and extending to the $900-1000-\mu \mathrm{m}$ size range. 
same size to better understand the impact of irregular shapes. Using computer-generated theoretical aggregates of hexagonal shapes as well as data from the CPI probe across a variety of conditions, we have shown that the standard deviation of $V_{t}$ calculated taking into account the observed variability in projected area and the assumed corresponding variability in mass within a given size bin is typically around $\pm 10 \%$ of the average $V_{t}$, with variability increasing in certain cases. More specifically, datasets that included large needle- and column-shaped crystals (Arctic and midlatitude wintertime cases) had higher variability when compared to purely convective cases. The greater differences were apparent at larger sizes $(>600 \mu \mathrm{m})$ and at higher temperatures $\left(>-15^{\circ} \mathrm{C}\right)$, although the data from highly convective field campaigns showed smaller or no changes with size or temperature. While the standard deviation typically suggests an envelope between $20 \%$ and $30 \%$ around the mean value in $V_{t}$, the implications could be substantially more given compounding effect of increased aggregation due to increased differential $V_{t}$ for a given ice particle population. The $V_{t}$ variability should be considered in modeling activities as it could promote increased aggregation opportunities because of increased differential sedimentation rates, even when narrow size distributions are predicted; moreover, the variability in $V_{t}$ for a given crystal size could affect the precipitation spatial distribution.

The inherent nature of randomness makes it difficult to determine an exact solution to these variabilities. As shown in Fig. 5, though, a FWHM value between 0.2 and 0.25 is reasonable for all regions and few adjustments by temperature or particle size are justified. Because of the nature of IPAS and the fractal analysis, the results presented here apply to complex particles or aggregates, and should not be assumed to be correct for individual ice crystals in the atmosphere. While the uncertainties in any of the individual calculations are small, the reader is cautioned that there are several layers of assumptions that go into the final result, and inaccuracies in the underlying assumptions could affect the uncertainty.

Acknowledgments. Funding for Schmitt, Sulia, Lebo, and Przybyo is through DOE Grant DE-SC0016354. Funding for ACTIVE and ACCACIA were from the Natural Environment Research Council, Grant NE/C512688/1 (ACTIVE) and NE/I028696/1 (ACCACIA), and data are available at http://badc.nerc.ac.uk/home/ index.html. EMERALD data are available from Connolly. CRYSTAL-FACE and MidCIX data are available https:// espoarchive.nasa.gov/archive/browse. ARM-IOP and MPACE data are available at http://archive.arm.gov. ICE-L, ICE-T, and AIRS2 data are available at https://www.eol.ucar.edu/ all-field-projects-and-deployments. The National Center for Atmospheric Research is sponsored by the National Science Foundation.

\section{REFERENCES}

Abdelmonem, A., M. Schnaiter, P. Amsler, E. Hesse, J. Meyer, and T. Leisner, 2011: First correlated measurements of the shape and light scattering properties of cloud particles using the new Particle Habit Imaging and Polar Scattering (PHIPS) probe. Atmos. Meas. Tech., 4, 2125-2142, https:// doi.org/10.5194/amt-4-2125-2011.

Brown, P. R. A., and P. N. Francis, 1995: Improved measurements of the ice water content of cirrus using an evaporative technique. J. Atmos. Oceanic Technol., 12, 410-414, https://doi.org/ 10.1175/1520-0426(1995)012<0410:IMOTIW>2.0.CO;2.

Cho, H.-R., J. V. Iribarne, and W. G. Richards, 1981: On the orientation of ice crystals in a cumulonimbus cloud. J. Atmos. Sci., 38, 1111-1114, https://doi.org/10.1175/1520-0469(1981) 038<1111:OTOOIC $>2.0 . \mathrm{CO} ; 2$.

Harrington, J. Y., K. J. Sulia, and H. Morrison, 2013a: A method for adaptive habit prediction in bulk microphysical models. Part II: Theoretical development. J. Atmos. Sci., 70, 349-364, https://doi.org/10.1175/JAS-D-12-040.1.

,-- , and,$- 2013 \mathrm{~b}$ : A method for adaptive habit prediction in bulk microphysical models. Part II: Parcel model corroboration. J. Atmos. Sci., 70, 365-376, https://doi.org/ 10.1175/JAS-D-12-0152.1.

Heymsfield, A. J., and L. M. Miloshevich, 2003: Parameterizations for the cross-sectional area and extinction of cirrus and stratiform ice cloud particles. J. Atmos. Sci., 60, 936-956, https:// doi.org/10.1175/1520-0469(2003)060<0936:PFTCSA > 2.0.CO;2.

— of ice particle fall speeds using laboratory and field measurements. J. Atmos. Sci., 67, 2469-2482, https://doi.org/10.1175/ 2010JAS3379.1.

_ , and P. Willis, 2014: Cloud conditions favoring secondary ice particle production in tropical maritime convection. J. Atmos. Sci., 71, 4500-4526, https://doi.org/10.1175/JAS-D-14-0093.1.

_- S. Lewis, A. Bansemer, J. Iaquinta, L. Milosovich, M. Kajikawa, C. Twohy, and M. Poellot, 2002: A general approach for deriving the properties of cirrus and stratiform ice cloud particles. J. Atmos. Sci., 59, 3-29, https://doi.org/10.1175/ 1520-0469(2002)059<0003:AGAFDT>2.0.CO;2.

- - A. Bansemer, C. Schmitt, C. Twohy, and M. R. Poellot, 2004: Effective ice particle densities derived from aircraft data. J. Atmos. Sci., 61, 982-1003, https://doi.org/10.1175/ 1520-0469(2004)061<0982:EIPDDF>2.0.CO;2.

—, C. Schmitt, A. Bansemer, G.-J. Zadelhoff, M. J. McGill, C. Twohy, and D. Baumgardner, 2006: Effective radius of ice cloud particle populations derived from aircraft probes. J. Atmos. Oceanic Technol., 23, 361-380, https://doi.org/ 10.1175/JTECH1857.1.

— , P. R. Field, M. Bailey, D. Rogers, J. Stith, C. Twohy, Z. Wang, and S. Haimov, 2011: Ice in clouds experimentLayer clouds. Part I: Ice growth rates derived from lenticular wave cloud penetrations. J. Atmos. Sci., 68, 2628-2654, https:// doi.org/10.1175/JAS-D-11-025.1.

_- C. Schmitt, and A. Bansemer, 2013: Ice cloud particle size distributions and pressure-dependent terminal velocities from in situ observations at temperatures from $0^{\circ}$ to $-86^{\circ} \mathrm{C}$. 
J. Atmos. Sci., 70, 4123-4154, https://doi.org/10.1175/JAS-D12-0124.1.

Isaac, G., and Coauthors, 2005: First results from the Alliance Icing Research Study II. 43rd Aerospace Sciences Meeting and Exhibit, American Institute of Aeronautics and Astronautics, Reno, NV, AIAA 2005-252, https://doi.org/10.2514/ 6.2005-252.

Jensen, A. A., J. Y. Harrington, H. Morrison, and J. A. Milbrant, 2017: Predicting ice shape evolution in a bulk microphysics model. J. Atmos. Sci., 74, 2081-2104, https://doi.org/10.1175/ JAS-D-16-0350.1.

Jensen, E. J., D. Starr, and O. B. Toon, 2004: Mission investigates tropical cirrus clouds. Eos, Trans. Amer. Geophys. Union, 85, 45-50, https://doi.org/10.1029/2004EO050002.

Korolev, A., and G. Isaac, 2003: Roundness and aspect ratio of particles in ice clouds. J. Atmos. Sci., 60, 1795-1808, https://doi.org/ 10.1175/1520-0469(2003)060<1795:RAAROP $>2.0 . C O ; 2$

Lawson, R. P., B. A. Baker, C. G. Schmitt, and T. L. Jensen, 2001: An overview of microphysical properties of Arctic clouds observed in May and July 1998 during FIRE ACE. J. Geophys. Res., 106, 14 989-15 014, https://doi.org/10.1029/ 2000JD900789.

— , D. O'Connor, P. Zmarzly, K. Weaver, B. A. Baker, Q. Mo, and H. Jonsson, 2006: The 2D-S (stereo) probe: Design and preliminary tests of a new airborne, high speed, highresolution particle imaging probe. J. Atmos. Oceanic Technol., 23, 1462-1477, https://doi.org/10.1175/JTECH1927.1.

Lloyd, G., and Coauthors, 2015: Observations and comparisons of cloud microphysical properties in spring and summertime Arctic stratocumulus clouds during the ACCACIA campaign. Atmos. Chem. Phys., 15, 3719-3737, https://doi.org/10.5194/ acp-15-3719-2015.

McFarquhar, G. M., J. Um, and R. Jackson, 2013: Small cloud particle shapes in mixed-phase clouds. J. Appl. Meteor. Climatol., 52, 1277-1293, https://doi.org/10.1175/JAMC-D-12-0114.1.

Mitchell, D. L., 1996: Use of mass- and area-dimensional power laws for determining precipitation particle terminal velocities. J. Atmos. Sci., 53, 1710-1723, https://doi.org/ 10.1175/1520-0469(1996)053<1710:UOMAAD > 2.0.CO;2.

Passarelli, R. E., and R. C. Srivastava, 1979: A new aspect of snowflake aggregation theory. J. Atmos. Sci., 36, 484-493, https://doi.org/ 10.1175/1520-0469(1979)036<0484:ANAOSA > 2.0.CO;2.

Przybylo, V. M., K. J. Sulia, C. G. Schmitt, Z. J. Lebo, and W. C. May, 2019: The Ice Particle and Aggregate Simulator (IPAS). Part I: To extract dimensional properties of ice-ice aggregates for microphysical parameterization. J. Atmos. Sci., 76, 1661-1676, https://doi.org/10.1175/JAS-D-18-0187.1.

Sasyo, Y., and T. Matsuo, 1980: On the statistical investigation of fall velocity of snowflakes. Pap. Meteor. Geophys., 31, 61-79, https://doi.org/10.2467/mripapers.31.61.

$\longrightarrow$, and - 1985: Effects of variations in falling velocities of snowflakes on their aggregation. J. Meteor. Soc. Japan, 63, 249-261, https://doi.org/10.2151/jmsj1965.63.2_249.

Schmitt, C. G., and A. J. Heymsfield, 2010: The dimensional characteristics of ice crystal aggregates from fractal geometry. J. Atmos. Sci., 67, 1605-1616, https://doi.org/10.1175/ 2009JAS3187.1.

— , and —-, 2014: Observational quantification of the separation of simple and complex atmospheric ice particles. Geophys. Res. Lett., 41, 1301-1307, https://doi.org/10.1002/ 2013GL058781.

— - — , P. Connolly, E. Järvinen, and M. Schnaiter, 2016: A global view of atmospheric ice particle complexity. Geophys. Res. Lett., 43, 11 913-11 920, https://doi.org/10.1002/ 2016GL071267.

Sulia, K., J. Y. Harrington, and H. Morrison, 2013: A method for adaptive habit prediction in bulk microphysical models. Part III: Applications and studies within a two-dimensional kinematic model. J. Atmos. Sci., 70, 3302-3320, https://doi.org/ 10.1175/JAS-D-12-0316.1.

Twohy, C. H., A. J. Schanot, and W. A. Cooper, 1997: Measurement of condensed water content in liquid and ice clouds using an airborne counterflow virtual impactor. J. Atmos. Oceanic Technol., 14, 197-202, https://doi.org/10.1175/1520-0426(1997) 014<0197:MOCWCI >2.0.CO;2.

Vaughan, G., C. Schiller, A. R. MacKenzie, K. Bower, T. Peter, H. Schlager, N. R. P. Harris, and P. T. May, 2008: SCOUTO3/ ACTIVE: High-altitude aircraft measurements around deep tropical convection. Bull. Amer. Meteor. Soc., 89, 647-662, https://doi.org/10.1175/BAMS-89-5-647.

Verlinde, J., and Coauthors, 2007: The Mixed-Phase Arctic Cloud Experiment. Bull. Amer. Meteor. Soc., 88, 205-221, https:// doi.org/10.1175/BAMS-88-2-205.

Whiteway, J., and Coauthors, 2004: Anatomy of cirrus clouds: Results from the Emerald airborne campaigns. Geophys. Res. Lett., 31, L24102, https://doi.org/10.1029/2004GL021201.

Wu, W., and G. M. McFarquhar, 2016: On the impacts of different definitions of maximum dimension for nonspherical particles recorded by 2D imaging probes. J. Atmos. Oceanic Technol., 33, 1057-1072, https://doi.org/10.1175/JTECH-D-15-0177.1. 